\title{
Evaluating Tag-Based Preference Obfuscation Systems
}

\author{
Andreas Pashalidis and Bart Preneel
}

\begin{abstract}
While personalisation is key to increase the usability of online services, disclosing one's preferences is undesirable from a privacy perspective, because it enables profiling through the linkage of what may otherwise be unlinkable service invocations. This paper considers an easily implementable class of obfuscation strategies as a means to mitigate these risks, and examines its privacy/utility tradeoff. Our results are based on simulations that take place within a modular evaluation framework that can seamlessly accommodate real-world data. We conducted experiments with different simulated behaviours and using two preference populations, namely a population of maximally diverse preferences and one consisting of the movie preferences of some Netflix users. We measure utility in a way that is specific to the application of preference obfuscation. Privacy is measured in terms of unlinkability, with respect to two different adversaries. Our results show that reasonable privacy/utility tradeoffs require the disclosure of only small amounts of preference information.
\end{abstract}

Index Terms-Preferences, Obfuscation, Privacy, Unlinkability.

\section{INTRODUCTION}

$\mathrm{K}^{\mathrm{r}}$ NOWING what a user likes or dislikes is a prerequisite for effective personalisation. Many service providers therefore gather and store the preferences of their users. However, since they do not share this data, user experience cannot be personalised consistently across services, and services that do not distinguish between users, such as services that are used only sporadically, do not benefit from automatic preferencebased personalisation at all. In order to address these shortcomings, certain pervasive computing systems (e.g. [1], [2]) provide a centralised server that, typically among other things, stores user preferences and automatically discloses them to services as needed. The Daidalos pervasive system [3] and Google's 'ads preference manager $^{1}$, for example, operate in this way; both systems gather and enable users to edit their preferences on a centralised server. The preferences are subsequently transmitted to other services in order to personalise the user's experience. In the case of Google ads, the user's preferences are used in order to select appropriate advertisements.

If extended accordingly, then certain 'user-centric' identity management systems, e.g. [4], [5], [6], also address the above shortcomings; an extension has to ensure that the system stores the user's preferences and discloses them to services just as it does with other attributes or credentials. While the users of services such as last.fm or delicious.com, for example, could benefit from such an approach, unfortunately current

- K.U. Leuven and IBBT, ESAT/SCD-COSIC, Kasteelpark Arenberg 10 , Leuven / Gaston Crommenlaan 8, Ghent-Ledeberg, Belgium.

E-mail: $\{$ andreas.pashalidis,bart.preneel\}@esat.kuleuven.be

1. www.google.com/ads/preferences/ services uniquely identify their users and store prefereces on the server side. Certain peer-to-peer systems, however, derive 'user interest profiles' on the user's device and anonymously share this information with other users in order to generate recommendations (see, for example, [7], [8]).

It is important to keep in mind that preferences are potentially identifying attributes; in fact, detailed preference information is so strongly identifying that it can be used for user authentication [9]. Due to the privacy concerns that are inherent in disclosing detailed preference data [10], the potential of preference-based personalisation is oftentimes not realised [11], and certain existing systems obfuscate preferences before releasing them to service providers (see, for example, [1], [2], [12]). The question thus arises how to best obfuscate preferences such that privacy is preserved as much as possible without destroying the personalisation potential.

In this paper, we make a first attempt to answer this question. In particular, we first identify and describe an easily implementable family of parameterised preference obfuscation strategies that operate in this setting. We then go ahead to analyse how effectively they protect privacy in terms of unlinkability as well as their impact on utility, with the goal to find default parametrisations that strike reasonable privacy/utility tradeoffs. It is important to keep in mind that some users are likely to take privacy more seriously than others (see, for example, [13]). Therefore, such defaults may neither lead to acceptable privacy nor utility for some users. Nevertheless, many users, in particular those that are less concerned about privacy, are presumably unlikely to change the defaults and will probably even be unaware of obfuscation in the first place.

Our results show that, very roughly speaking, achieving reasonable privacy/utility tradeoffs requires the dis- 
closure of only small amounts of preference information, e.g. disclosing only two or three 'tags' which are each time randomly chosen from the user's five most preferred ones. The optimal default obfuscation parameters, however, depend to a large extent on details of the operational context, such as the behaviour of users, applicable privacy thresholds, and the distribution of preferences over the user population. There are, therefore, no definitive answers on which are 'the best' default obfuscation parameters. The main contribution of this paper is the evaluation framework and methodology itself.

The rest of this paper is organised as follows. After briefly surveying related work in the next section, Section 3 describes how we model preferences and their obfuscation, the obfuscation strategies we consider, the system model, and the adversary model. Section 4 describes two adversaries on unlinkability that make use of Bayesian inference and Section 5 introduces our modular simulation framework. Sections 6 and 7 show and discusses some of our results and Section 8 concludes.

\section{Related Work}

The present work relates to several research areas, as outlined below.

Privacy-preserving recommender systems: The most closely related works from this area are, perhaps, the studies by McSherry and Mironov [14] and Shokri and Pedarsani [15]. While the former enhances an existing centralised recommender system with provisions for differential privacy [16], the latter relies on a peer-topeer protocol according to which users discover how to best modify their own preference in order to make it look similar to the preferences of other users. Both [14] and [15] require cross-linking of preferences; while in [14] a central server performs this task, in [15], the users perform the task in a distributed manner. The approach taken in this paper does not require any cross-linking of preferences, and this frees users from the risks of trusting a central server, and Sibyl attacks [17]. This freedom, however, comes at the cost of not being able to exploit explicit knowledge of the distribution of preferences, which may have an impact on the achieved privacy/utility tradeoffs. Our results and algorithms are not directly comparable to the ones used in [14], [15] due to their different modelling of preferences.

Utility-based anonymisation: The focus of utility-based anonymisation systems is the tradeoff between privacy and utility. Naturally, different applications require different utility measures [18], and existing systems are based on a variety of utility measures that, typically, quantify the loss of information in some general sense (see, for example, [10], [19], [20], [21], [22], [23]). In this paper we do not use a generic utility measure; in order to directly capture the potential effectiveness of personalisation, we define a measure based on the similarity between real and obfuscated preferences. The most closely related work from this field is, perhaps, the approach proposed by Krause and Horvitz [10]. Its main difference with our work lies in the fact that, [10] aims to identify the optimal subset of user attributes to disclose to a service while, in this paper we aim to identify the optimal obfuscation for preferences.

Privacy definitions and metrics: Various measurable notions of privacy have been defined in the context of privacy-preserving systems (e.g. [24], [25], [26], [27], [28], [29], [30]) and, in particular, data randomisation systems (e.g. [16], [31], [32]). One natural approach to define privacy in our setting, based on ideas from $k$ anonymity [21], would be to divide users with similar preferences into sufficiently large groups (i.e. of size at least $k$ ), and then disclose only obfuscated preferences that prevent distinguishing users within their respective group, e.g. simply by using a single 'representative' preference for the entire group. Unfortunately, this approach again requires the cross-linking of preferences [18]. In this paper, we use the measure defined in [33] in order to evaluate how closely the adversary's 'guess' approximates the true clustering. The measure supports more fine-grained evaluations than previously proposed measures (e.g. [34], [35], [36]) and essentially computes a number $s \in[0,1]$ that represents the risk that the adversary manages to link a given percentage - the 'privacy threshold' - of a user's service invocations; this notion of a privacy threshold enables us to examine the effectiveness of obfuscation along this additional dimension.

Clustering: Our adversaries are relatively simple clustering algorithms that, at their core, use Bayesian inference [37]. While certainly not the only one, the Bayesian approach has been used in similar contexts (for example by An, Jutla and Cercone [38] and by Linamoodand and Kantarcioglu [39]). In contrast to other popular clustering algorithms such as k-means and most hierarchical and probabilistic techniques, our algorithms do not require an external determination of the number of clusters that should be produced. On the other hand, our adversaries produce a single guess rather than a probability distribution over the solution space. Our first adversary is an efficient greedy algorithm while our second adversary makes use of simulated annealing. Simulated annealing-based clustering was first introduced by Klein and Dubes [40] and Selim and Alsultan [41], and recently used in a privacy context by Schiffner and Clauß [42]. These works, too, assume that the number of clusters is known; our adversaries therefore explore an exponentially (in the number of transactions) larger solution space (see section 3.2 of [35]). For an overview of clustering techniques the reader is referred to the survey by Delgrande et al. [43], [44].

Representation of preferences: We represent preferences as permutations (i.e. rankings) of a fixed set of tags. This representation was chosen because it seems to suffice for personalisation in many applications while omitting unnecessary details. While it is common [45], [46], [47], [48], [49] (and tags are used by popular web services 
such as StumbleUpon, Google AdSense and various social bookmarking services), alternative representations are sometimes used, typically depending on how preferences are acquired. Some systems, for example, construct the user's preference as a total order from a history of explicit positive or negative ratings, that user issued over tagged content. ${ }^{2}$ Certain machine learning and data mining techniques, on the other hand, represent preferences as partial orders [50], [51], [52]. It is, however, typically straight-forward to convert alternative preference representations into a total order (i.e. a permutation or ranking), for example by resolving ties arbitrarily or by rounding [23]. Of course, converting representations that operate over individual items of a large set may involve first extracting some features (tags) and subsequently clustering the set of items. For a survey of different preference representations the reader is referred to [53]. Other related work: Recently, machine learning techniques have been used to attack privacy-preserving systems [54], [55]. While these works focus on different applications and types of obfuscation, they are similar in spirit to our work. In fact, their results together with ours demonstrate that machine learning provides a means to evaluate obfuscation systems in general. Another marginally related research area is that of private information retrieval (PIR) [56]. The goal of PIR systems is to enable a client to retrieve a data item from the server without the server learning which exactly item was retrieved. A 'PIR-like' approach in our setting would require the server to send all its content to the client, and the client to render the content based on the user's preference. While this approach is certainly more privacy-friendly (as no preference data ever leaves the client), it is beyond our scope since (a) it requires the client to be able to render user interfaces for multiple applications, and (b) is not applicable in the setting where a centralised server pushes preferences to services on the user's behalf.

\section{MOdEL AND NOTATION}

This section describes how we model preferences and their obfuscation as well as the interactions of users within the system, and the adversary model. The notation, summarised in the table below, is introduced along with the description.

\subsection{Preferences and their obfuscation}

We model preferences as rankings over a finite set of tags. If, for example, different cuisines are tagged using the set of tags $T=\{$ 'Italian', 'French', 'Indian', 'Mexican', 'Japanese', 'Thai' $\}$, then a preference could be, for example, the ranking $p=$ ('French', 'Thai', 'Mexican', 'Italian', 'Japanese', 'Indian'). Formally, a preference is a permutation $p=\left(t_{1}, t_{2}, \ldots, t_{|T|}\right) \in M(T)$, where $T$

2. Typically, tags that have not yet been rated will be placed 'between' liked and disliked tags in some way. is a finite set of tags, $M(T)$ denotes the set of all its permutations, and the ordering reflects the preference, most preferred first.

We denote by $T\left(p, p^{\prime}, \pi\right) \subseteq T$ the set of tags that is represented in the first $\pi$ positions in both the preferences $p=\left(t_{1}, t_{2}, \ldots, t_{|T|}\right)$ and $p^{\prime}=\left(t_{1}^{\prime}, t_{2}^{\prime}, \ldots, t_{|T|}^{\prime}\right)$. That is, for all $1 \leq \pi \leq|T|, T\left(p, p^{\prime}, \pi\right)=\left\{t_{1}, t_{2}, \ldots, t_{\pi}\right\} \cap$ $\left\{t_{1}^{\prime}, t_{2}^{\prime}, \ldots, t_{\pi}^{\prime}\right\}$. Let $\Omega=\{\omega: M(T) \rightarrow \Delta(M(T))\}$, where $\Delta(M(T))$ is the set of all probability distributions over $M(T)$, denote the space of functions that, on input a preference, output a probability distribution over $M(T)$. A preference obfuscation strategy $\sigma$ is a randomised process that takes as input a preference and returns another preference. If the output of an obfuscation strategy is chosen according to an obfuscation function $\omega \in \Omega$, then the strategy is said to apply $\omega$, and is denoted by $\sigma_{\omega}$. The output space of an obfuscation strategy $\sigma_{\omega}$ with respect to a preference $p$, denoted $\left\{\sigma_{\omega}(p)\right\}$, is the set of preferences that have positive probability of being the obfuscated version of $p$ according to $\sigma_{\omega}$. That is, $\left\{\sigma_{\omega}(p)\right\}=\left\{p^{\prime} \in M(T): \operatorname{Pr}\left(\sigma_{\omega}(p)=p^{\prime}\right)>0\right\}$. An obfuscation strategy $\sigma_{\omega}$ is said to have constant output size if, for all $p, p^{\prime} \in M(T),\left|\left\{\sigma_{\omega}(p)\right\}\right|=\left|\left\{\sigma_{\omega}\left(p^{\prime}\right)\right\}\right|$, and is said to be uniform if, for all $p \in M(T)$ and all $p^{\prime} \in\{\omega(p)\}$, $\operatorname{Pr}\left(\sigma_{\omega}(p)=p^{\prime}\right)=\left|\left\{\sigma_{\omega}(p)\right\}\right|^{-1}$.

\begin{tabular}{|l|l|}
\hline Symbol & Meaning \\
\hline$T, t$ & Set of tags, tag \\
$M(T)$ & Set of all tag permutations \\
$p, p^{\prime} \in M(T)$ & real preference, obfuscated preference \\
$T(\cdot, \cdot, \cdot)$ & function that returns tag subset \\
$\kappa, \lambda(\kappa \leq \lambda)$ & obfuscation parameters \\
$n$ & total number of service invocations \\
$\mathcal{N}$ & true partition of service invocations \\
$\mathcal{N}^{\prime}$ & adversary's best guess for $\mathcal{N}$ \\
$u=|\mathcal{N}|$ & number of users in the system \\
$\beta$ & privacy threshold, defined in [33] \\
$s(\mathcal{A}, \beta)$ & adversary's success degree (sect. 5.3.1) \\
$a(\mathcal{A}, \beta)$ & adversary's advantage (sect. 5.3.1) \\
$\mathrm{r} \in\{1, \mathrm{e}\}$ & indicator for utility variant (sect. 5.3.2) \\
$\mathcal{Q}^{\mathrm{r}}$ & overall system utility (sect. 5.3.2) \\
$F$ & objective function (sect. 5.3.3) \\
\hline
\end{tabular}

\subsection{The $(\kappa, \lambda)$-family of obfuscation strategies}

In this paper, we focus on a particular family of obfuscation strategies which we call the family of $(\kappa, \lambda)$ strategies. It was chosen due to its intuitive parametrisation, and the fact that it is very easily and efficiently implementable and relatively easy to analyse. All members of this family are uniform with constant output size, and perform obfuscation as follows. First, the input ('real') preference is divided into two groups, namely the group of favoured, and the group of unfavoured tags. Then, the tags in each group are permuted independently and uniformly at random. Finally, the obfuscated preference is constructed by appending the (permuted) unfavoured tags to the (permuted) favoured ones. 
The parameters $\kappa$ and $\lambda$, which must be such that $0 \leq$ $\kappa \leq \lambda<|T|$, specify how the groups of favourable and unfavourable tags are constructed: the set of favourable tags, which is selected first, is determined by uniformly at random selecting $\kappa$ out of the first $\lambda$ tags of the input preference; the group of unfavourable tags is the set of the remaining $|T|-\kappa$ tags. Fig. 1 shows an obfuscation example with parameters $(\kappa, \lambda)=(2,3)$ where the second and the third tag happen to get selected as the set of favourable tags. Formally, $\omega^{(\kappa, \lambda)}(p)$ is the obfuscation function that, on input $p=\left(t_{1}, t_{2}, \ldots, t_{|T|}\right)$, outputs the probability distribution where, for all $p^{\prime}=$ $\left(t_{1}^{\prime}, t_{2}^{\prime}, \ldots, t_{|T|}^{\prime}\right) \in M(T)$,

$$
\operatorname{Pr}\left(p^{\prime}\right)= \begin{cases}\frac{(\lambda-\kappa) !}{\lambda !(|T|-\kappa) !}, & \text { if }\left\{t_{1}^{\prime}, \ldots, t_{\kappa}^{\prime}\right\} \subseteq\left\{t_{1}, \ldots, t_{\lambda}\right\} \\ 0, & \text { otherwise. }\end{cases}
$$

\section{Fig. 1. Obfuscation example}

Remark 1 It suffices to transmit the first $\kappa$ tags instead of the entire obfuscated preference. While this reduces communication overhead (see Remark 4), care has to be taken in the case where obfuscation parameters differ from user to user (this case is not considered in this paper). This is because different values for $\kappa$ enable linking on the basis of these values in this case.

\subsection{System model}

We consider the setting where a set of $u$ users invokes one or more services via an anonymous communication channel. We denote the total number of service invocations by $n$. Each user has a preference that, for the sake of simplicity, is assumed to be static. ${ }^{3}$ We denote the user preferences by $p_{1}, p_{2}, \ldots, p_{u}$. Every time a user invokes a service, his preference gets obfuscated by an obfuscation strategy, and the resulting obfuscated preference is disclosed to the service. We assume that the same strategy $\sigma_{\omega(\kappa, \lambda)}$ is applied to all users and service invocations.

As a result of the service invocations, a sequence $p_{1}^{\prime}, p_{2}^{\prime}, \ldots, p_{n}^{\prime} \in M(T)$ of obfuscated preferences is produced, where, for all $i \in\{1,2, \ldots, n\}, p_{i}^{\prime}=\sigma_{\omega^{(\kappa, \lambda)}}\left(p_{j}\right)$ for some $p_{j} \in\left\{p_{1}, p_{2}, \ldots, p_{u}\right\}$. Consider the 'origin' function $o:\{1,2, \ldots, n\} \rightarrow\left\{p_{1}, p_{2}, \ldots, p_{u}\right\}$ that maps the serial numbers of service invocations (i.e. the indices of obfuscated preferences) to the real preferences of the users. We denote by $\mathcal{N}=\left\{N_{1}, N_{2}, \ldots, N_{u}\right\}$ the partition that $o$ induces on $\{1,2, \ldots, n\}$. The notation $i \sim i^{\prime}$ means that invocations $i$ and $i^{\prime}$ originate from the same user, i.e. that $\left\{i, i^{\prime}\right\} \subseteq N$ for some $N \in \mathcal{N}$.

3. Our adversaries can cope with a certain amount of variation in the user preferences. It is merely required that the set of favourable and unfavourable tags (as determined by the obfuscation parameters) remains static, while the order of tags within each subset may change.

\subsection{Adversary model}

We consider the case of a coalition of services that follows the system's protocols, but that attempts to violate the privacy of users by trying to link service invocations originating from the same user. The adversary's input consists of publicly known information about the obfuscation process in the system, and information about the distribution of preferences over users. In particular, its input consists of the sequence $p_{1}^{\prime}, p_{2}^{\prime}, \ldots, p_{n}^{\prime}$ of obfuscated preferences, the probability distribution according to which 'real' preferences are distributed over the users, and the obfuscation parameters $\kappa, \lambda$.

The adversary's goal is to link the service invocations, i.e. to identify $\mathcal{N}$ by dividing the set $\{1,2, \ldots, n\}$ of service invocation serial numbers into clusters such that each cluster contains the invocations that correspond to a single user.

We emphasize that the adversary's goal is not to 'deanonymise' users. This is because our focus is to identify optimal obfuscation parameters; for this, it seems appropriate to limit the adversary such that it exclusively obtains the information leaked by insufficient obfuscation (rather than by other data fields that may or may not be present in a deployment and that would make a de-anonymisation attack possible). In our model, the adversary is assumed to know nothing about the behaviour of the users. This implies that it knows neither the sizes, nor the number $u$ of users (i.e. the number of equivalence classes in $\mathcal{N}$ ).

The adversary's goal can be considered as a step towards de-obfuscation of users' preferences. This is because, if the adversary has successfully collected all obfuscated versions of given user's preference, then filtering out the remaining noise that was added by the obfuscation algorithm is likely to reveal a preference that is very similar or even identical to the user's real preference. We do not, however, focus on de-obfuscation since constructing the user's transaction history is typically more intrusive than reconstructing his true preference.

\section{LINKING SERVICE INVOCATIONS}

In order to link service invocations on the basis of obfuscated preferences, intuition suggests that the adversary may restrict its attention to the set of favourable tags, i.e. the first $\kappa$ tags in each obfuscated preference, since all information is contained in this set. Based on this observation, and within the adversarial model described in section 3.4, we construct in this section two adversaries that focus on the favourable tags that obfuscated preferences have in common. Note that, if $\lambda>\kappa$, then two obfuscated preferences may originate from the same original preference even if fewer than their first $\kappa$ tags match; if $\kappa<\lambda / 2$, then two obfuscated versions of the same preference may not even have any of their first $\kappa$ tags in common.

More precisely, for all pairs of obfuscated preferences $\left(p_{i}^{\prime}, p_{i^{\prime}}^{\prime}\right)$, our adversaries observe $T\left(p_{i}^{\prime}, p_{i^{\prime}}^{\prime}, \kappa\right)$, i.e. the fa- 
vorable tags that they have in common; we denote the event that $T\left(p_{i}^{\prime}, p_{i^{\prime}}^{\prime}, \kappa\right)=T^{\prime}$ for some $T^{\prime} \subseteq T$ by $E_{T^{\prime}}$ or simply by $E$ if $T^{\prime}$ is clear from the context. Moreover, the adversaries make use of a 'Bayesian oracle' that operates as follows.

Bayesian oracle: Consider any two distinct invocations $0 \leq i<i^{\prime} \leq n$, and let the hypothesis $H$ be the event that $i \sim i^{\prime}$. Given the prior probability $\operatorname{Pr}(H)$ as well as the conditional probabilities $\operatorname{Pr}\left(E_{T^{\prime}} \mid H\right)$ and $\operatorname{Pr}\left(E_{T^{\prime}} \mid \bar{H}\right)$ for some observed evidence $E_{T^{\prime}}$, the Bayesian oracle simply applies Bayes' rule [57] $\operatorname{Pr}\left(H \mid E_{T^{\prime}}\right)=$ $\operatorname{Pr}\left(E_{T^{\prime}} \mid H\right) \operatorname{Pr}(H) /\left(\operatorname{Pr}\left(E_{T^{\prime}} \mid H\right) \operatorname{Pr}(H)+\operatorname{Pr}\left(E_{T^{\prime}} \mid \bar{H}\right) \operatorname{Pr}(\bar{H})\right)$ and returns the posterior probability that $H$ is true. Note that the prior probability $\operatorname{Pr}(H)$ as well as the conditional probabilities $\operatorname{Pr}\left(E_{T^{\prime}} \mid H\right)$, and $\operatorname{Pr}\left(E_{T^{\prime}} \mid \bar{H}\right)$ depend on how preferences are distributed over the users and on the behaviour of users, and this varies from application to application. Section 5 examines two different cases.

\subsection{Clustering}

We consider two clustering algorithms, based on optimisation techniques, namely 'hill climbing' (or simple greedy algorithm), and simulated annealing. Both algorithms output a single guess $\mathcal{N}^{\prime} \in L(\{1,2, \ldots, n\})$ for $\mathcal{N}$, where $L(\cdot)$ denotes the set of partitions (clusterings, equivalence relations) of a set. The greedy algorithm, shown in Fig. 2, starts with the partition of singletons and greedily merges clusters up to a certain point. Its complexity is $O\left(n^{2}\right)$. Our simulated annealing-based clustering algorithm, shown in Fig. 3, on the other hand, starts with a random candidate guess. As is standard in simulated annealing, it explores the solution space by moving to a 'better' candidate whenever it finds one, but also moves to a 'worse' candidate sometimes in order to avoid being stuck in local optima. The algorithm constructs each of a given candidate's neighbours in step 2(a) by first choosing a tag at random, and then by reallocating this tag from its current cluster to another (possibly empty) cluster. In order to work, the algorithm must be able to assess how good any given candidate partition $C \in L(1,2, \ldots, n)$ appears to be; we use the objective function

$$
\begin{aligned}
G(C)= & \sum_{\substack{\left\{i, i^{\prime}\right\} \subseteq\{1,2, \ldots, n\} \\
\exists N \in C:\left\{i, i^{\prime}\right\} \subseteq N}} \operatorname{Pr}\left(i \sim i^{\prime} \mid E\right)+ \\
& \sum_{\substack{\left\{i, i^{\prime}\right\} \subseteq\{1,2, \ldots, n\} \\
\nexists N \in C:\left\{i, i^{\prime}\right\} \subseteq N}}\left(1-\operatorname{Pr}\left(i \sim i^{\prime} \mid E\right)\right),
\end{aligned}
$$

where the values for $\operatorname{Pr}\left(i \sim i^{\prime} \mid E\right)$ come from the Bayesian oracle. The algorithm's running time is dominated by the 'time budget' $t=\alpha \log _{2}\left(B_{n}\right)$ where $B_{n}$ is the $n$th Bell number [58] (which is the size of the solution space from which $\mathcal{N}^{\prime}$ is drawn [35]), and $\alpha$ is a coefficient whose value we determine experimentally in section 6 .
Greedy Adversary (input: $\kappa, \lambda, p_{1}^{\prime}, p_{2}^{\prime}, \ldots, p_{n}^{\prime}$ ):

1) Use the Bayesian oracle to calculate $\operatorname{Pr}\left(i \sim i^{\prime} \mid E\right)$ for all $i, i^{\prime} \in\{1,2, \ldots, n\}$.

2) Start with $\mathcal{N}^{\prime}=\{\{1\},\{2\}, \ldots,\{n\}\}$.

3) Select $\left\{N, N^{\prime}\right\} \subseteq \mathcal{N}^{\prime}$ such that

$$
\delta=\prod_{\substack{i \in N \\ i^{\prime} \in N^{\prime}}} \operatorname{Pr}\left(i \sim i^{\prime} \mid E\right)-\prod_{\substack{i \in N \\ i^{\prime} \in N^{\prime}}}\left(1-\operatorname{Pr}\left(i \sim i^{\prime} \mid E\right)\right)
$$

is maximised.

4) While $\delta>0$ do the following.

a) Merge $N$ and $N^{\prime}$ and update $\mathcal{N}^{\prime}$.

b) Update $N, N^{\prime}$, and $\delta$ as in step 3 .

5) Output $\mathcal{N}^{\prime}$.

Fig. 2. Clustering by hill climbing

SA Adversary (input: $\left.\alpha, \kappa, \lambda, p_{1}^{\prime}, p_{2}^{\prime}, \ldots, p_{n}^{\prime}\right)$ :

1) Start with a random $S \in L(\{1,2, \ldots, n\})$, and set $\mathcal{N}^{\prime}=S, t=\alpha \log _{2}\left(B_{n}\right), s=2 / t$, and $h=1$.

2) While $t>0$ do the following.

a) Select a neighbour $C$ of $\mathcal{N}^{\prime}$, at random.

b) If $G(C)>G\left(\mathcal{N}^{\prime}\right)$, then set $\mathcal{N}^{\prime}=C$.

c) If $G(C)>G(S)$, then set $S=C$. Otherwise

i) Choose a random $r \in[0,1]$.

ii) If $r<h$, then set $S=C$.

d) Decrease $t$ by one and $h$ by $s$.

3) Output $S$.

Fig. 3. Clustering by simulated annealing

Since $\log _{2}\left(B_{n}\right)$ grows roughly linearly ${ }^{4}$, and since the evaluation of Eq. (2) takes quadratic time in $n$, the algorithm's complexity is roughly $O\left(n^{3}\right)$. Note that the algorithm becomes greedy (i.e. no longer moves to worse solutions) during the second half of its time budget.

\section{EXPERIMENTAL SETUP}

At the centre of our modular simulation framework, outlined in Fig. 4, is an experiment runner which, in order to run a single experiment, invokes the instance generator on input the set of tags $T$, the desired length of the sequence of obfuscated preferences $(n)$, and the obfuscation parameters $\kappa, \lambda$ (step 1 in the figure). The instance generator then simulates the users and outputs a sequence $p_{1}^{\prime}, p_{2}^{\prime}, \ldots, p_{n}^{\prime}$ of obfuscated preferences as well as the solution $\mathcal{N}$ (step 2). The experiment runner then invokes a series of preconfigured adversaries on input the sequence of obfuscated preferences (step 3). Each adversary outputs a single guess for $\mathcal{N}$ (step 4) which is then picked up by the experiment runner and compared to $\mathcal{N}$ (step 5).

The instance generator is divided into three different modules, as follows. On input $n$, the user behaviour module chooses the solution $\mathcal{N}$ by simulating user behaviour,

4. See [59, p.108] for a more accurate asymptotic formula for $\log _{2}\left(B_{n}\right)$. 


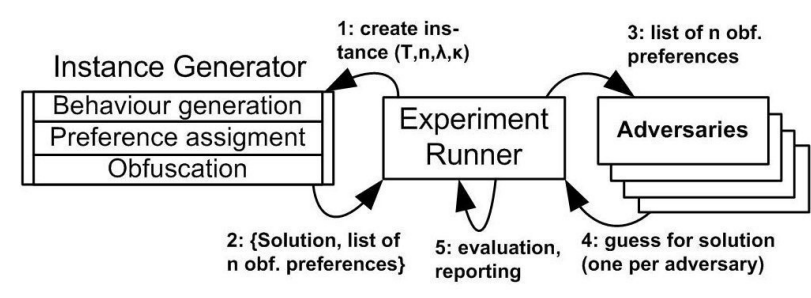

Fig. 4. Modular simulation framework

i.e. by determining which users invoke the service in which order. Note that, as a 'side effect' of choosing $\mathcal{N}$, the user behaviour module also chooses the number $u=|\mathcal{N}|$ of users in the system. On input $u$ and $T$, the preference assignment module assigns, to each of the $u$ users, a 'real' preference from $M(T)$. Finally, on input the solution $\mathcal{N}$ and the $u$ real preferences, the obfuscation module produces the sequence of obfuscated preferences simply by iteratively applying the obfuscation strategy $\sigma_{\omega(\kappa, \lambda)}$.

\subsection{Behaviour generation}

Since we do not have access to real behaviour data, our behaviour generation modules generate artifical behaviours. We emphasize that it is unclear whether or not the generated behaviours resemble realistic user behaviour. Our main motivation, however, is to test the robustness of our adversaries, i.e. the extent to which they can cope with different behaviours. Since our adversaries know nothing about user behaviour, they assume, a priori, that every possible solution is equally likely. That is, we take the a priori probability of the hypothesis being true to be $\operatorname{Pr}(H)=\Pi(n, 2)$, where $\Pi(\cdot, \cdot)$ is given in Lemma 6 (see Appendix B). We conducted experiments with three user behaviour modules, which we describe below.

Unlinkable behaviour. This module, denoted UB, produces behaviours such that all solutions are equally likely; it chooses $\mathcal{N}$ uniformly at random from $L(\{1,2, \ldots, n\})$. (For an efficient way to do this, see chapter 10 of [60].) That is, this module produces behaviours that actually do correspond to the adversaries' prior view. We note that this method of behaviour generation guarantees that any adversary is restricted to make use of the information that is leaked exclusively by obfuscated preferences (rather than a potential tendency to prefer solutions that are biased towards more 'typical' user behaviours).

Non-uniform behaviour. This module, denoted NB, simulates $n$ invocations as follows. The initial user population consists of a single user who performs the first invocation. For each subsequent invocation, a fair coin is tossed. In case of heads, a new user performs the invocation and is added to the population; otherwise, a user already in the population is selected uniformly at random and performs the invocation.
Poisson process behaviour. This module, denoted $P B$, simulates a population of $3 n$ users, each using an exponential distribution in order to select the next point in time at which he will invoke the service. The means for these distributions were selected according to a Zipf distribution with parameter $a=2.5$, using the algorithm described in [61, p.551]. The simulation stops after the first $n$ invocations have taken place, at which point of course only few (maximally $n$, but typically less) users will have interacted.

Remark 2 The NB and PB modules generate behaviours that induce probabilities over the solution space $L(\{1,2, \ldots, n\})$ that significantly differ from the uniform distribution.

\subsection{Preference assignment}

We use two different preference assignment methods in our experiments. The next subsections describe these methods and provide the resulting formulas for the conditional probabilities $\operatorname{Pr}\left(E_{T^{\prime}} \mid H\right), \operatorname{Pr}\left(E_{T^{\prime}} \mid \bar{H}\right)$, and $\operatorname{Pr}\left(E_{\pi} \mid \bar{H}\right)$.

\subsubsection{Uniform preference assignment}

This module assigns preferences to users by drawing $u$ preferences uniformly at random from $M(T)$. This models the setting where the tags in $T$ are uncorrelated, i.e. are selected in a manner that leads to an optimally compact preference encoding with respect to the user population. Selecting tags in this manner makes sense since personalisation is most effective when user preferences differ as much as possible. However, we do not expect such an encoding to become widespread in practice; if tags are specified manually (as in a 'folksonomy' [62]), then selecting tags in this manner requires one to know the user preferences beforehand, and this is unrealistic. In machine learning (automatic inference), tags may be periodically redefined with respect to the user population, and then user preferences over the new tag set can be computed, e.g. from collected ratings [63] or implicit feedback [64]. Perhaps a uniform distribution can be approximated if tags are post-processed by means of principal component analysis [65].

While such considerations are beyond the scope of this paper, it remains important to study this preference assignment method because it minimises the probability of users having the same or similar preferences and hence represents the worst case from a privacy perspective; any particular obfuscation parameters that yield a particular privacy level in this setting are unlikely to result in worse levels in other settings. We also note that this preference assignment method follows the 'random worlds' paradigm [66] and is similar in spirit with studies from the area of network traffic analysis (e.g. [67]). The following lemma, proven in Appendix $C$, provides the required formulas for the conditional probabilities $\operatorname{Pr}\left(E_{T^{\prime}} \mid H\right)$ and $\operatorname{Pr}\left(E_{T^{\prime}} \mid \bar{H}\right)$. 


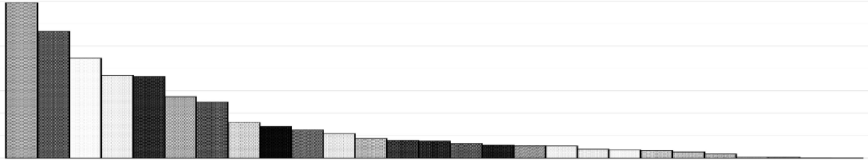

Fig. 5. Relative tag popularity of Netflix users. Observe that the distribution is very far from uniform.

Lemma 1 Given the obfuscation strategy $\sigma_{\omega^{(\kappa, \lambda)}}$ and the preference assignment module described above, the probabilities that $T\left(p_{i}^{\prime}, p_{i^{\prime}}^{\prime}, \kappa\right)=T^{\prime}$ conditioned on the events that $i \sim i^{\prime}$ and $i \neq i^{\prime}$ are, for all $T^{\prime} \subseteq T$, given by

$$
\begin{gathered}
\operatorname{Pr}\left(E_{T^{\prime}} \mid H\right)=\left(\begin{array}{c}
\lambda-\left|T^{\prime}\right| \\
\kappa-\left|T^{\prime}\right|
\end{array}\right)\left(\begin{array}{c}
\lambda-\kappa \\
\kappa-\left|T^{\prime}\right|
\end{array}\right)\left(\begin{array}{c}
|T|-\left|T^{\prime}\right| \\
\lambda-\left|T^{\prime}\right|
\end{array}\right)\left(\begin{array}{c}
|T| \\
\lambda
\end{array}\right)^{-1}\left(\begin{array}{c}
\lambda \\
\kappa
\end{array}\right)^{-2} \text { and } \\
\operatorname{Pr}\left(E_{T^{\prime}} \mid \bar{H}\right)=\left(\begin{array}{c}
|T|-\kappa \\
\kappa-\left|T^{\prime}\right|
\end{array}\right)\left(\begin{array}{c}
|T|-\left|T^{\prime}\right| \\
\kappa-\left|T^{\prime}\right|
\end{array}\right)\left(\begin{array}{c}
|T| \\
\kappa
\end{array}\right)^{-2} \text {, respectively. }
\end{gathered}
$$

\subsubsection{Netflix preference assignment}

For this module, we derived a 'population of preferences' as follows. First, we tagged the movies in the Netflix data set [68] according to the 27 genre tags from the Internet Movies Database (IMDB) 'The Mummy', for example, was assigned the tags 'Action', 'Adventure', 'Comedy', 'Fantasy', 'Horror', and 'Thriller'. Only a part of the Netflix movies was tagged because they had to be matched against the IMDB based only on title and year of production and, in order to avoid potential misclassifications, the matching rules we applied were rather strict; out of the 17,770 movies in the Netflix dataset, only 10,440 were tagged. It turned out that 479,617 users had rated these movies and, based on their ratings, the preference of each of these users was constructed, as follows. Each user was assigned 27 counters (one for each possible tag) and, for all his ratings $r$, the tag counters corresponding to the tags assigned to the movie in question were increased by $r$. The straightforward ranking of the tags according to the final counter values (largest value first) was taken as the final preference. Fig. 5 shows the relative popularity of tags over the entire set of users; note that the distribution of preferences is very far from being uniform.

The Netflix preference assignment module operates simply by selecting the $u$ preferences from a given preference population $P$ using simple random sampling with replacement. The preference population we provided to the module was the one derived from the Netflix data set as described above. The following lemmas, proven in Appendix $\mathrm{C}$, provide the required formulas for conditional probabilities $\operatorname{Pr}\left(E_{T^{\prime}} \mid H\right)$ and $\operatorname{Pr}\left(E_{T^{\prime}} \mid \bar{H}\right)$.

Lemma 2 Given the obfuscation strategy $\sigma_{\omega^{(\kappa, \lambda)}}$, a preference population $P$ and the preference assignment module described above, the probabilities that $T\left(p_{i}^{\prime}, p_{i^{\prime}}^{\prime}, \kappa\right)=T^{\prime}$, conditioned on the events that $i \sim i^{\prime}$ and $i \neq i^{\prime}$, is, for all $T^{\prime} \subseteq T$, given by

5. http://www. imdb.com

$$
\begin{aligned}
& \operatorname{Pr}\left(E_{T^{\prime}} \mid H\right)= \Gamma_{\mathrm{p}}\left(P, \lambda, T^{\prime}\right)\left(\begin{array}{c}
\lambda-\kappa \\
\kappa-\left|T^{\prime}\right|
\end{array}\right)\left(\begin{array}{l}
\lambda-\left|T^{\prime}\right| \\
\kappa-\left|T^{\prime}\right|
\end{array}\right)\left(\begin{array}{l}
\lambda \\
\kappa
\end{array}\right)^{-2} \text { and } \quad(5) \\
& \operatorname{Pr}\left(E_{T^{\prime}} \mid \bar{H}\right)=\left(\begin{array}{c}
\lambda \\
\kappa
\end{array}\right)-2 \sum_{\substack{T_{c} \subseteq T-T^{\prime} \\
\left|T_{c}\right|=\kappa-\left|T^{\prime}\right|}} \Gamma_{\mathrm{p}}\left(P, \lambda, T^{\prime} \cup T_{c}\right) \\
& \sum_{T_{x} \subseteq T_{c}}(-1)^{\left|T_{x}\right|} \Gamma_{\mathrm{p}}\left(P, \lambda, T^{\prime} \cup T_{x}\right)\left(\begin{array}{c}
\lambda-\left|T^{\prime}\right|-\left|T_{x}\right| \\
\kappa-\left|T^{\prime}\right|
\end{array}\right),
\end{aligned}
$$

respectively, and where $\Gamma_{\mathrm{p}}(\cdot, \cdot, \cdot)$ is given by Eq. (11) (see Appendix $A$ ).

Remark 3 The evaluation of Eq. (6) takes, in the worst case, i.e. in the case where $T^{\prime}=\emptyset,\left(\begin{array}{c}|T| \\ \kappa\end{array}\right) 2^{\kappa}$ steps. For example, if $\kappa=4$ and $T$ contains 27 tags (which is the case for the data derived from the Netflix data set), then the evaluation of Eq. (6) may take up to 280, 800 steps. Since Netflix users' preferences are, however, similar (see Fig. 5), the worst case occurs only rarely. The values of $\Gamma_{\mathrm{p}}(\cdot, \cdot, \cdot)$ can be precomputed.

\subsection{Evaluation}

In each of our experiments, the experiment runner records the utility and the degree of privacy achieved during the experiment, as well as the time each adversary took until outputting its guess. The relevant measures are described below.

\subsubsection{Privacy measure}

The system's privacy in face of an adversary $\mathcal{A}$ is measured in terms of how well $\mathcal{A}^{\prime}$ s output $\mathcal{N}^{\prime}$ approximates the solution $\mathcal{N}$. More precisely, we use the "secondary errors' measure proposed in [33]. This measure requires two additional parameters, as follows. Firstly, the parameter $\alpha \in[0,1]$ indicates the relative importance of the adversary's ability to link a given user's invocations, versus its ability to keep a user's invocations separate from the invocations of other users. We attach the same importance to both abilities in all our evaluations. That is, we set $\alpha=1 / 2$ and we will henceforth omit mentioning this parameter. The second parameter, $\beta \in[0,1]$, is the fraction of a user's invocations that the adversary should not be able to link. When evaluated with a particular value for $\beta$, the measure returns a 'risk level' that indicates how close the adversary's guess actually is to breaching the given threshold. We apply the same privacy threshold to all users, and subsequently calculate the measure for the average user, again using the same privacy threshold, as described in [33].

We call the result of an evaluation of the privacy measure, obtained as described above, $\mathcal{A}^{\prime}$ s success degree and denote it by $s(\mathcal{A}, \beta)$. Moreover, $\mathcal{A}$ 's advantage over random guessing is defined as $a(\mathcal{A}, \beta)=s(\mathcal{A}, \beta)-s(\mathrm{RP}, \beta)$ where RP denotes a dummy algorithm that simply outputs a partition of $\{1,2, \ldots, n\}$, chosen uniformly at random. 


\subsubsection{Utility metrics}

We use a distance metric on permutations as the basis to measure utility. The intuition is that, as the distance of the obfuscated preference from the user's real preference increases, the personalisation potential decreases. Our distance metric is based on the number of times one has to swap adjacent elements in one permutation in order to arrive at another [69], [70]. However, our metric is also weighted such that more weight is given to the user's more preferred tags, and less to his less preferred ones. This captures the intuition that preserving the position of the user's most preferred tags leads to more effective personalisation than preserving the position of less preferred tags.

Hence, the position of a tag in the preference determines the weight it carries; tags in higher positions carry less weight than tags in lower positions. The exact value of a tag's weight depends on the weight assigned to the first tag, and the rate at which weights decrease. We consider two utility metrics, namely one where the weight decreases linearly, and one where it decreases exponentially fast. In particular, the 'linear' weight of a tag at position $\nu \in\{1,2, \ldots,|T|\}$ is given by $w_{\nu}^{1}=1-(\nu /|T|)$, and its '(negative) exponential' weight by $w_{\nu}^{\mathrm{e}}=2^{-\nu-1}$. Based on this, we define the linearly and the negative exponentially weighted distance between two permutations $p^{\prime}=\left(t_{1}^{\prime}, t_{2}^{\prime}, \ldots, t_{|T|}^{\prime}\right)$ and $p=\left(t_{1}, t_{2}, \ldots, t_{|T|}\right)$ as

$$
\eta^{\mathrm{r}}\left(p^{\prime}, p\right)=\sum_{\mu=1}^{\left|p^{\prime}\right|} \sum_{\nu=\mu+1}^{\left|p^{\prime}\right|} x\left(p^{\prime}, p, \mu, \nu\right)\left(w_{\nu}^{\mathrm{r}}+w_{\mu}^{\mathrm{r}}\right),
$$

where $r \in\{l, e\}$ denotes the rate at which preference weights decrease, and where $x\left(p^{\prime}, p, \mu, \nu\right)=0$ if $\left(t_{\mu}^{\prime}, t_{\nu}^{\prime}\right)$ appear in the same order in both $p^{\prime}$ and $p$, and $x\left(p^{\prime}, p, \mu, \nu\right)=1$ otherwise. Note that $\eta^{\mathrm{r}}\left(p^{\prime}, p\right)=0$ if and only if $p^{\prime}=p$ and that the maximum distance $\max _{\eta^{\mathrm{r}}}\left(p^{\prime}, p\right)=\sum_{\mu=1}^{\left|p^{\prime}\right|} \sum_{\nu=\mu+1}^{\left|p^{\prime}\right|}\left(w_{\nu}^{\mathrm{r}}+w_{\mu}^{\mathrm{r}}\right)$ is reached if and only if $x\left(p^{\prime}, p, \mu, \nu\right)=1$ for all $1 \leq \mu<\nu \leq\left|p^{\prime}\right|$, i.e. when $p^{\prime}$ is the reversal of $p$. For a comparison to other distance metrics on permutations the reader is referred to [71]. The utility of a service invocation $i \in\{1,2, \ldots, n\}$ is defined as $q^{\mathrm{r}}(i)=1-\eta^{\mathrm{r}}\left(p_{i}^{\prime}, p_{o(i)}\right) / \max _{\eta^{\mathrm{r}}}\left(p_{i}^{\prime}, p_{o(i)}\right)$. Note that $q^{\mathrm{r}}(i)$ takes values in $[0,1]$ with $q^{\mathrm{r}}(i)=1$ if and only if $p^{\prime}=p$ and $q^{\mathrm{r}}(i)=0$ if and only if $p^{\prime}$ is the reversal of $p$. Finally, the average utility experienced by the user with real preference $p_{j} \in\left\{p_{1}, p_{2}, \ldots, p_{u}\right\}$ is given by $Q^{\mathrm{r}}\left(p_{j}\right)=\sum_{i \in N_{j}} q^{\mathrm{r}}(i) /\left|N_{j}\right|$ and the overall system utility by $\mathcal{Q}^{\mathrm{r}}=\sum_{j} Q^{\mathrm{r}}(j) / u$.

Remark 4 If $|T|$ is large, then there is little value in transmitting the entire preference to a service, because the exact order of the least preferred tags is rather insignificant in terms of personalisation (also see Remark 1).

\subsubsection{Privacy/utility tradeoff}

The privacy gain of an obfuscation strategy has to be balanced against the utility loss it induces. We use the objective function $F=\mathcal{Q}^{\mathrm{r}}-\max _{\beta} a(\mathcal{A}, \beta)$ as the basis to optimise the tradeoff between utility and privacy, where $r \in\{1, e\}$ indicates whether the linear or the exponential utility function is used. $F$ takes values between -1 and 1 , and our task is to find obfuscation parameters $\kappa, \lambda$ that maximise $F$. The maximising combination, however, may depend not only on the preference assignment method and user behaviour, but also on $n$, i.e. on how many service invocations the adversary aims to link. In order to determine the optimal obfuscation parameters with respect to an entire range of values for $n$, we choose some maximum $\lambda_{\mu}$ and $n_{\mu}$, and perform experiments for all parameter combinations such that $\lambda \in\left\{1,2, \ldots, \lambda_{\mu}\right\}$ and $\kappa \leq \lambda$, and repeat these experiments for each value of $n \in\left\{2,3, \ldots, n_{\mu}\right\}$. Then we rank the parameter combinations by placing the $(\kappa, \lambda)$-combination yielding the highest value for $F$ in the first position, the one yielding the second highest value for $F$ in the second position, and so on. In the end, a cumulative score is assigned to each considered $(\kappa, \lambda)$-combination as

$$
\mathbf{s c}_{\kappa, \lambda}=\sum_{n=2}^{n_{\mu}}\left[1-\left(\operatorname{pos}_{\kappa, \lambda, n} / c\right)\right]
$$

where $\operatorname{pos}_{\kappa, \lambda, n}$ denotes the rank of the $(\kappa, \lambda)$ combination for the given value of $n$, and $c=\lambda_{\mu}\left(\lambda_{\mu}+3\right) / 2$ denotes the total number of combinations considered for that value of $n$.

\section{Results}

All plots show the averages of 1000 experiment repetitions. The simulated annealing-based adversary is denoted by SAA, and the greedy adversary by GRA, and the dummy algorithm that outputs a random partition by RP. We determined the value of the coefficient $\alpha$ (see section 4.1) for SAA experimentally; our results show that values of $\alpha$ greater than 30 do not yield any significant increase in the adversary's success degree. Thus, in our experiments, we set $\alpha=30$.

Fig. 6 shows how the number of to-be-linked invocations $n$ affects the success degrees of GRA and SAA, where obfuscation parameters were fixed at $(\kappa, \lambda)=(4,5)$, and the privacy threshold was taken to be $\beta=80 \%$. Given that GRA and SAA explore the solution space in fundamentally different ways, we found it surprising that their success degree is quite similar. Moreover, our experiments show that, despite the fact that it explores a smaller fraction of the solution space, even for different privacy thresholds, GRA is at least slightly more successful than SAA.

Fig. 7 highlights the importance of examining the entire spectrum of possible privacy thresholds in face of an attack. It shows how the threshold $\beta$ affects the advantages of our adversaries (without distinguishing between GRA and SAA), for all $n \in\{30, \ldots, 75\}$. The reason we excluded values below 30 is because the shown trends start to manifest themselves above this value. The plots show that, in the uniform preference 


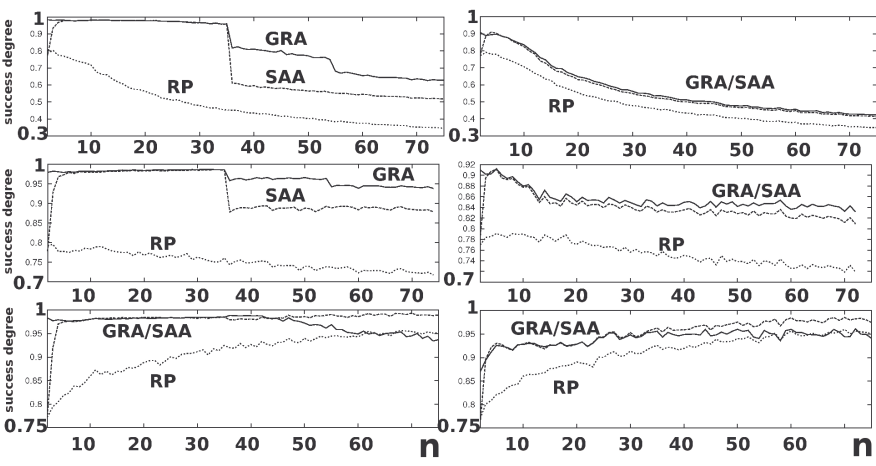

Fig. 6. Success degrees $s(\mathcal{A}, 0.8)$ of our two adversaries $\mathrm{SAA}$ and GRA and the dummy algorithm RP, as a function of $n$. Graphs on the left and the right refer to the uniform and Netflix preference assignment case, respectively, and the first (resp. second, third) row shows results where user behaviour was generated using the UB (resp. NB, PB) module.
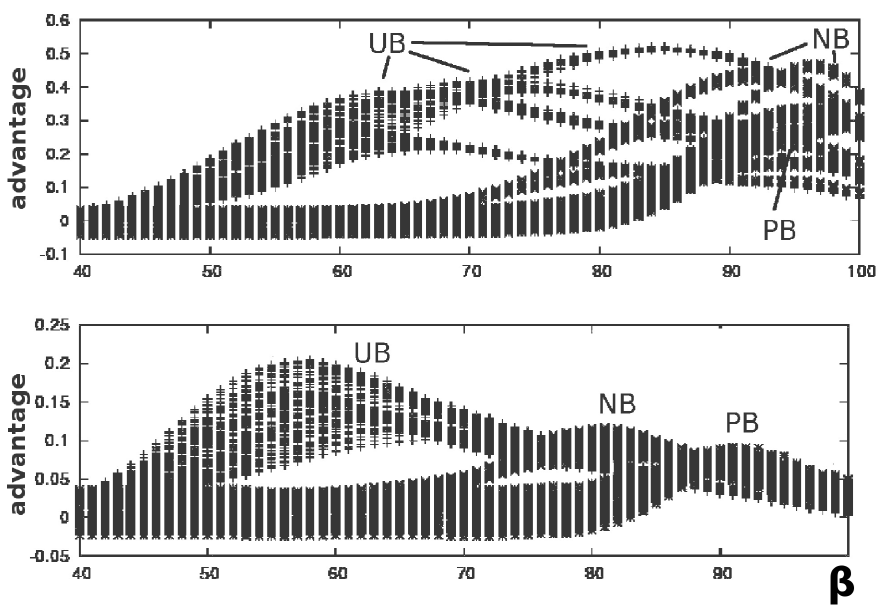

Fig. 7. Advantages $a(\mathrm{GRA}, \beta)$ and $a(\mathrm{SAA}, \beta)$ as a function of $\beta$, for all $\beta \in[0.4,1]$ and for all $n \in\{30,31, \ldots, 75\}$. Each value of $n$ results in a different curve (see text).

assignment case, the advantage is maximised for values of $60 \%-85 \%, 85 \%-95 \%$, and around $95 \%-98 \%$ for UB, $\mathrm{NB}$, and $\mathrm{PB}$ behaviour, respectively, where the exact value of $\beta$ that maximises the advantage depends on $n$. In the Netflix case, this dependence on $n$ is significantly weaker since the corresponding ranges are shorter: $55 \%-62 \%$, $78 \%-83 \%$, and $90 \%-95 \%$.

Fig. 8 shows the top five parameter combinations

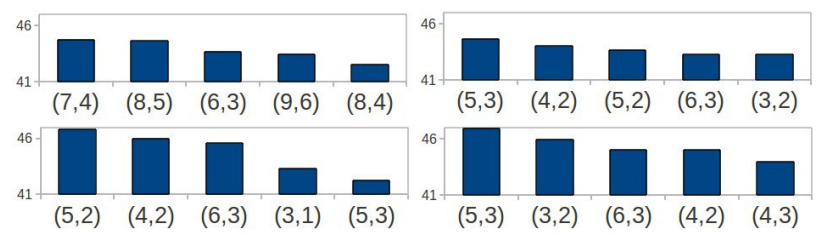

Fig. 8. Top five $(\kappa, \lambda)$-parameter combinations in various settings along with their score (see Eq. (7) and text).

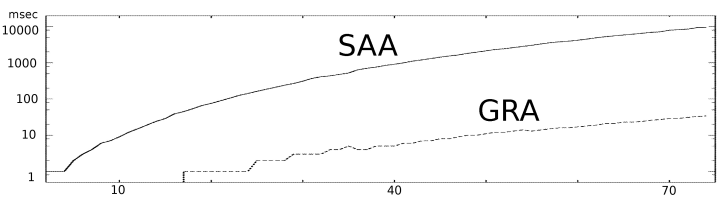

Fig. 9. Running time of our adversaries as a function of $n$

along with their score (see Eq. (7)), based on a large number of experiments, where $\lambda_{\mu}=9, n_{\mu}=50$, and each experiment was repeated 1000 times in order to establish the GRA's average advantage (for speed reasons, we did not consider SAA in this experiment set). Results on the left and right refer to the uniform and the Netflix case, respectively, and the upper and lower rows show the results for the linear and exponential utility measures.

The results for the uniform case show the effect of the choice of the utility measure. Observe that, under the exponential variant, four top combinations are such that $\kappa \leq \lambda / 2$, while, in the linear variant, this is the case for only two combinations. In other words, choosing the exponential variant implies that optimal obfuscation implies heavier obfuscation. Somewhat surprisingly, this is not true in the Netflix case, where the same three parameter combinations are present in the top five for both utility variants.

Finally, Fig. 9 shows the running time of our adversaries on a computer with a $3 \mathrm{GHz}$ processor. When trying to link 75 invocations $(n=75)$, for example, the SAA (with $\alpha=30$ ) takes more than 10 seconds to come up with a guess, while GRA outputs its guess in less than 20 milliseconds. The preference assignment method (Netflix or uniform) has almost no effect on the running time. Our dummy algorithm RP outputs a random partition in maximally a couple of milliseconds for all $n \leq 75$.

\section{Discussion}

In most cases, the success degree of our adversaries decreases as $n$ grows. $^{6}$ Except for $\mathrm{PB}$ behaviour, this is not true for their advantage, as the success degree of the dummy algorithm decreases at least as fast; is most cases, the adversaries' advantage remains positive even for large values of $n$ (e.g. $n \approx 1000$ ). Nevertheless, as $n$ grows, the dependence of the adversaries' success degree on user behaviour increases, too. This means that, for the adversary it makes sense to filter out as many invocations as possible, and focus its attack only on those invocations that it is interested in.

The advantage of our adversaries also depends on user behaviour and, at least for some underlying privacy

6. This is not surprising since a larger $n$ generally implies a larger number of users and an exponentially larger solution space. Hence, the possibilities of error as captured by the unlinkability measure increase dramatically; this can be seen by the decrease of success of RP. An exception occurs for PB behaviour since this behaviour generates few large (rather than many small) clusters (i.e. typically only few users are active). This limits the error possibilities causing higher linkability measurements. 
thresholds and in all three examined user behaviour models, our adversaries yielded significant advantages. This shows that our chosen adversaries' prior view is relatively robust, i.e. that the fact that they are assumed to know nothing about user behaviour generally leads to correct inferences. This is a reassuring result since it suggests that our adversaries are likely to exhibit a significant advantage also when confronted with real user behaviour. However, the differences in advantage between our adversaries were also significant (see Fig. 7). This suggests that their success degrees and advantage are likely to increase if the prior probabilities are adjusted to the underlying scenario.

It is important to keep in mind that different user behaviours induce different 'vulnerable' privacy thresholds, as shown in Fig. 7. Thus, when confronted with real user behaviour, users with certain privacy thresholds will be more exposed than others. We have based our objective function $F$ on the adversary's advantage for the most vulnerable privacy threshold. While serving the privacy of all users, this can also be seen as a weakness, since, for users with less vulnerable thresholds, the optimal obfuscation parameters may to lead to unnecessarily low levels of utility. However, since defaults apply to all users, we choose to err in favour of those users with the most vulnerable privacy thresholds.

The strongest dependence of our adversaries' success degrees is on the preference assignment method. This is no surprise since they extract information about the target clustering based on the values of obfuscated preferences; the more dissimilar the preferences of different users, the more such information there is; while, for example, the probability that 3 out of the 5 most favoured tags of two users are identical is approximately $26.5 \%$ in the uniform case (with $|T|=12$ ), in the Netflix case this probability is approximately $73 \%$ (for the tags 'Drama', 'Comedy', and 'Thriller'). As a consequence, success degrees are considerably higher in the uniform preference assignment case than they are in the Netflix case.

In general, our results suggest that, for optimal and near-optimal obfuscation parameter choices, $\kappa \approx \lambda / 2$. That is, disclosing roughly $\lambda / 2$ tags, which are each time randomly chosen from one's $\lambda$ most favourable tags and then randomly reordered, is likely to lead to a reasonable privacy/utility tradeoff.

It should be stressed that for a real, deployed system with known user behaviour patterns, the simulations should be re-run with a user behaviour generation module that respects these patterns. It should also be stressed that the adversaries we consider in this paper merely observe the favourable tags that two obfuscated preferences have in common. That is, they ignore the favourable tags in which two obfuscated preferences differ - and this last piece of information is likely to contain additional information especially under heavy obfuscation (i.e. when $\kappa<\lambda / 2$ ). Also, while they know nothing about the number and behaviour of users, such knowledge (i.e. adjusting the adversaries' prior views to the underlying application) may to lead to higher success degrees in some scenarios. Moreover, while our adversaries use a greedy and a simulated annealing strategy to search the solution space, other clustering techniques, or techniques that make use of different optimisation and cluster similarity functions, may similarly lead to good success degrees.

Hence we believe that our attacks can be improved without excessively strengthening the adversary model. This means that derived obfuscation parameters should not be interpreted as being in any generic way optimal (as they are so only with respect to the considered adversaries and measures), but rather as a lower bound; while heavier obfuscation levels are likely to strike acceptable tradeoffs, less heavy ones are not.

Should we therefore conclude that preference obfuscation based on permutations generally fails? Not necessarily, as the family of obfuscation strategies we considered makes no assumptions on, and therefore does not exploit any knowledge of, the distribution of preferences over users. We believe that exploiting statistical information about this distribution is likely to lead to permutationbased obfuscation strategies (not members of the family considered in this paper) that are more resistant to our and similar linkability attacks. While such strategies do not need to rely on runtime cross-linking of preferences, they assume that statistical information is available, and hence that cross-linking has occurred in the past at least once. Identifying and evaluating such strategies is an important future research direction.

We note that our modular simulation framework can seamlessly accommodate new obfuscation strategies, new adversaries and different measures; thus, the derivation of new results is a straight-forward task, and the main difficulty lies in the definition and implementation of the new adversaries.

\section{Conclusions}

We presented a class of preference obfuscation strategies that operate on a typical preference encoding (namely a ranking over tags), that does not require the preferences of different users to be cross-linked. The strategies apply both in the setting where users independently provide their preferences to service providers, and where a centralised party takes over this task. We furthermore examined the privacy/utility tradeoff that the obfuscation strategies yield, with the aim to find optimal obfuscation parameters. In order to do this, we constructed a number of adversaries that try to link service invocations on the basis of obfuscated preferences.

Our experiments took place in a modular simulation framework, and the results reveal the dependencies of our adversaries' success degree and advantage on the number of to-be-linked invocations, the behaviour of users, and distribution of preferences as well as applicable privacy thresholds. Our results show that these 
dependencies are, in general, significant. Moreover, our adversaries are limited in several respects. Nevertheless, we believe that it is safe to say that, typically, only significant amounts of obfuscation, such as when one randomly picks roughly two of one's five most favourable tags, and then presents these tags in a random order as his preference, can justify the utility gained from disclosing the preference.

Future work items include the development of adversaries that also observe differing favourable tags and have various degrees of background knowledge about user behaviour, and examining the case where obfuscation parameters vary from user to user. Of course, if users with atypical preferences obfuscate more heavily than users with typical ones, then better privacy/utility tradeoffs seem to be possible [15]. This assumes, however, that users are made aware of how typical their preference really is, possibly via cross-linking of preferences, and achieving this in a privacy-preserving manner is not trivial.

We also intend to extend our simulation framework for measuring unlinkability in other settings such as electronic cash and, more generally, attribute exchange. Such an extension would ideally encompass a more powerful Bayesian oracle that operates over arbitrary subsets of 'items of interest' rather than merely pairs, as well as other clustering algorithms.

\section{ACKNOWLEDGMENTS}

The authors are grateful to Jens-Matthias Bohli, Kajetan Dolinar, Mario Lischka, Markulf Kohlweiss, Stefan Schiffner, Carmela Troncoso and Frederik Vercauteren; their help and suggestions significantly increased the quality of this paper. This paper describes work undertaken partly in the context of the 'Trusted Architecture for Securely Shared Services' (TAS3) project (www.tas3.eu) (TAS3 is a collaborative project supported by the 7th European Framework Programme, with contract number 216287), partly by the IAP Programme P6/26 BCRYPT of the Belgian State (Belgian Science Policy), and partly by the Research Council K.U.Leuven: GOA TENSE.

\section{REFERENCES}

[1] F. L. Gandon and N. M. Sadeh, "Semantic web technologies to reconcile privacy and context awareness," Web Semantics: Science, Services and Agents on the World Wide Web, vol. 1, no. 3, pp. 241260, 2004.

[2] D. Riboni, L. Pareschi, and C. Bettini, "Privacy in georeferenced context-aware services: A survey," in Proceedings of the First International Workshop on Privacy in Location-Based Applications (PiLBA '08), October 9, 2008 - Malaga, Spain, C. Bettini, S. Jajodia, P. Samarati, and X. S. Wang, Eds., 2008.

[3] N. Taylor, P. Robertson, B. Farshchian, K. Doolin, I. Roussaki, L. Marshall, R. Mullins, S. Druesedow, and K. Dolinar, "Pervasive computing in daidalos," IEEE Pervasive Computing, vol. 10, pp. 74-81, January 2011. [Online]. Available: http://dx.doi.org/10.1109/MPRV.2010.24
[4] J. Camenisch and E. Van Herreweghen, "Design and implementation of the idemix anonymous credential system," in Proceedings of the 9th ACM conference on Computer and Communications Security, 2002, pp. 21-30.

[5] K. Cameron and M. B. Jones, "Design rationale behind the identity metasystem architecture," in ISSE/SECURE 2007 Securing Electronic Business Processes. Vieweg, 2007, pp. 117-129.

[6] S. Clauß, D. Kesdogan, and T. Kölsch, "Privacy enhancing identity management: protection against re-identification and profiling," in Proceedings of the 2005 workshop on Digital Identity Management. New York, NY, USA: ACM Press, 2005, pp. 84-93.

[7] J. A. Pouwelse, P. Garbacki, J. Wang, A. Bakker, J. Yang, A. Iosup, D. H. J. Epema, M. Reinders, M. R. van Steen, and H. J. Sips, "Tribler: a social-based peer-to-peer system," Concurrency and Computation: Practice and Experience, vol. 20, no. 2, pp. 127-138, 2008. [Online]. Available: http://dx.doi.org/10.1002/cpe.1189

[8] M. Waaijers, J. Wang, J. A. Pouwelse, J. Fokker, A. P. de Vries, and M. J. T. Reinders, "Personalization On A Peer-To-Peer Television System," International Journal on Multimedia Tools and Applications, vol. 36, no. 1-2, pp. 89 - 113, January 2008.

[9] M. Jakobsson, E. Stolterman, S. Wetzel, and L. Yang, "Love and authentication," in Proceedings of the 26th Annual Conference on Human Factors in Computing Systems (CHI 2008), April 2008, pp. 197-200.

[10] A. Krause and E. Horvitz, "A utility-theoretic approach to privacy and personalization," in Proceedings of AAAI-08: Twenty-Third Conference on Artificial Intelligence.

[11] R. K. Chellappa and R. G. Sin, "Personalization versus privacy: An empirical examination of the online consumers dilemma," Information Technology and Management, vol. 6, no. 2-3, pp. 181202, May 2005.

[12] R. Wishart, K. Henricksen, and J. Indulska, "Context privacy and obfuscation supported by dynamic context source discovery and processing in a context management system," in Ubiquitous Intelligence and Computing, ser. Lecture Notes in Computer Science. Springer Verlag, Berlin, 2007, no. 4611, pp. 929-940.

[13] A. Acquisti and J. Grossklags, "Privacy and rationality in individual decision making," IEEE Security and Privacy, vol. 3, pp. 26-33, January 2005. [Online]. Available: http:/ / portal.acm.org/ citation.cfm?id=1048715.1048819

[14] F. McSherry and I. Mironov, "Differentially private recommender systems: building privacy into the net," in KDD '09: Proceedings of the 15th ACM SIGKDD international conference on Knowledge discovery and data mining. New York, NY, USA: ACM, 2009, pp. 627-636.

[15] R. Shokri, P. Pedarsani, G. Theodorakopoulos, and J.-P. Hubaux "Preserving Privacy in Collaborative Filtering through Distributed Aggregation of Offline Profiles," in The 3rd ACM Conference on Recommender Systems (RecSys). ACM, 2009.

[16] M. Bugliesi, B. Preneel, V. Sassone, and I. Wegener, Eds., Differential Privacy, ser. Lecture Notes in Computer Science, vol. 4052. Springer, 2006.

[17] J. Douceur, "The sybil attack," in Peer-to-Peer Systems, First International Workshop, IPTPS 2002, Cambridge, MA, USA, March 7-8, 2002, Revised Papers, ser. Lecture Notes in Computer Science, P. Druschel, F. Kaashoek, and A. Rowstron, Eds., no. 2429. Springer Verlag, Berlin, 2002, pp. 251-260.

[18] C. C. Aggarwal and P. S. Yu, "A general survey of privacypreserving data mining models and algorithms," in PrivacyPreserving Data Mining: Models and Algorithms, ser. Advannces in Database Systems, C. C. Aggarwal and P. S. Yu, Eds. Springer Verlag, Berlin, 2008, no. 34, ch. 2, pp. 11-52.

[19] V. S. Iyengar, "Transforming data to satisfy privacy constraints," in Proceedings of the eighth ACM SIGKDD international conference on Knowledge discovery and data mining. ACM Press, 2002, pp. 279-288.

[20] P. Samarati, "Protecting respondents identities in microdata release," IEEE Transactions on Knowledge and Data Engineering, vol. 13, no. 6, pp. 1010-1027, 2001.

[21] P. Samarati and L. Sweeney, "Protecting privacy when disclosing information: k-anonymity and its enforcement through generalization and suppression," Computer Science Laboratory, SRI International, Tech. Rep. SRI-CSL-98-04, 1998.

[22] L. Sweeney, "Datafly: A system for providing anonymity in medical data," in Proceedings of the IFIP TC11 WG11.3 Eleventh International Conference on Database Securty XI: Status and Prospects, 
ser. IFIP Conference Proceedings, vol. 113. Chapman \& Hall, Ltd. London, UK, 1997, pp. 356-381.

[23] L. Willenborg and T. DeWaal, Elements of Statistical Disclosure Control. Springer Verlag, New York, 2001.

[24] S. Clauß and S. Schiffner, "Structuring anonymity metrics," in DIM '06: Proceedings of the second ACM workshop on Digital identity management. New York, NY, USA: ACM Press, 2006, pp. 55-62.

[25] C. Díaz, "Anonymity metrics revisited," in Anonymous Communication and its Applications, number 05411 in Dagstuhl Seminar Proceedings, 2005.

[26] C. Díaz, S. Seys, J. Claessens, and B. Preneel, "Towards measuring anonymity," in Privacy Enhancing Technologies, Second International Workshop, PET 2002, San Francisco, CA, USA, April 14-15, 2002, Revised Papers, ser. Lecture Notes in Computer Science, R. Dingledine and P. F. Syverson, Eds., no. 2482. Springer Verlag, Berlin, 2002, pp. 54-68.

[27] M. Edman, F. Sivrikaya, and B. Yener, "A combinatorial approach to measuring anonymity," in Proceedings of the 2007 IEEE International Conference on Intelligence and Security Informatics. IEEE, 2007.

[28] B. Gierlichs, C. Troncoso, C. Díaz, B. Preneel, and I. Verbauwhede, "Revisiting a combinatorial approach toward measuring anonymity," in Proceedings of the 2008 ACM Workshop on Privacy in the Electronic Society, WPES 2008, Alexandria, Virginia, USA, October 27, 2008. ACM Press, 2008, pp. 111-116.

[29] V. Shmatikov and M.-H. Wang, "Measuring relationship anonymity in mix networks," in WPES '06: Proceedings of the 5th ACM workshop on Privacy in electronic society. New York, NY, USA: ACM Press, 2006, pp. 59-62.

[30] G. Tóth, Z. Hornák, and F. Vajda, "Measuring anonymity revisited," in Proceedings of the Ninth Nordic Workshop on Secure IT Systems, S. Liimatainen and T. Virtanen, Eds., Espoo, Finland, November 2004, pp. 85-90.

[31] D. Agrawal and C. C. Aggarwal, "On the design and quantification of privacy preserving data mining algorithms," in Proceedings of the Twentieth ACM SIGACT-SIGMOD-SIGART Symposium on Principles of Database Systems. Santa Barbara, California, USA. May 21-23 2001. ACM Press, 2001.

[32] R. Agrawal and R. Srikant, "Privacy-preserving data mining," in Proc. of the ACM SIGMOD Conference on Management of Data, 2000, pp. 439-450.

[33] A. Pashalidis and S. Schiffner, "Evaluating adversarial partitions," in European Symposium on Research in Computer Security, ESORICS 2010, Proceedings, ser. Lecture Notes in Computer Science, D. Gritzalis and B. Preneel, Eds., no. 6345. Springer Verlag, Berlin, 2010, pp. 524-539.

[34] L. Fischer, S. Katzenbeisser, and C. Eckert, "Measuring unlinkability revisited," in Proceedings of the 2008 ACM Workshop on Privacy in the Electronic Society, WPES 2008, Alexandria, Virginia, USA, October 27, 2008. ACM Press, 2008, pp. 111-116.

[35] M. Franz, B. Meyer, and A. Pashalidis, "Attacking unlinkability: The importance of context," in Privacy Enhancing Technologies, 7th International Symposium, PET 2007 Ottawa, Canada, June 2022, 2007, Revised Selected Papers, ser. Lecture Notes in Computer Science, N. Borisov and P. Golle, Eds., vol. 4776. Springer Verlag, Berlin, 2007, pp. 1-16.

[36] A. Pashalidis, "Measuring the effectiveness and the fairness of relation hiding systems," in Proceedings of the First International Workshop on Multimedia, Information Privacy and Intelligent Computing Systems, 2008.

[37] T. Hastie, R. Tibshirani, and J. Friedman, The Elements of Statistical Learning: Data Mining, Inference, and Prediction, 2nd ed., ser. Springer Series in Statistics. Springer Verlag, Berlin, December 2008.

[38] X. An, D. Jutla, and N. Cercone, "Temporal context lie detection and generation," in Secure Data Management, ser. Lecture Notes in Computer Science, vol. 4165. Springer Verlag, Berlin, 2006, pp. $30-47$.

[39] J. Lindamoodand and M. Kantarcioglu, "Inferring private information using social network data," University of Texas at Dallas, Tech. Rep. UTDCS-29-08, July 2008.

[40] R. W. Klein and R. C. Dubes, "Experiments in projection and clustering by simulated annealing," Pattern Recognition, vol. 22, no. 2, pp. 213-220, 1989.

[41] S. E. Selim and K. Alsultan, "A simulated annealing algorithm for the clustering problem," Pattern Recognition, vol. 24, no. 10, pp. 1003-1008, 1991.
[42] S. Schiffner and S. Clauß, "Using linkability information to attack mix-based anonymity services," in Privacy Enhancing Technologies, 9th International Symposium, PETS 2009, Seattle, WA, USA, August 5-7, 2009. Proceedings.

[43] I. H. Witten and E. Frank, Data Mining: Practical Machine Learning Tools and Techniques, 2nd ed., ser. Morgan Kaufmann series in data management systems. Morgan Kaufmann, June 2005.

[44] R. Xu and D. Wunsch, Clustering. Wiley-IEEE Press, October 2008.

[45] W. W. Cohen, R. E. Schapire, and Y. Singer, "Learning to order things," in Advances in Neural Information Processing Systems 10, [NIPS Conference,Denver, Colorado, USA, 1997].

[46] M. desJardins, E. Eaton, and K. L. Wagstaff, "Learning user preferences for sets of objects," in Proceedings of the 23rd international conference on Machine learning, vol. 148. ACM Press, 2006, pp. 273-280.

[47] J. Fürnkranz and E. Hüllermeier, "Pairwise preference learning and ranking," in Machine Learning: ECML 2003, 14th European Conference on Machine Learning, Cavtat-Dubrovnik, Croatia, September 22-26, 2003, Proceedings, ser. Lecture Notes in Computer Science, N. Lavrac, D. Gamberger, L. Todorovski, and H. Blockeel, Eds., vol. 2837. Springer Verlag, Berlin, 2003, pp. 145-156.

[48] — , "Preference learning," KI, 2005.

[49] K. Hindriks, C. Jonker, and W. Visser, "Reasoning about multiattribute preferences (short paper)," in AAMAS '09: Proceedings of The 8th International Conference on Autonomous Agents and Multiagent Systems.

[50] W. W. Cohen, R. E. Schapire, and Y. Singer, "Learning to order things," Journal of Artificial Intelligence Research, vol. 10, pp. 243$270,1999$.

[51] S. Holland, M. Ester, and W. Kießling, "Preference mining: A novel approach on mining user preferences for personalized applications," in Knowledge Discovery in Databases: PKDD 2003, ser. Lecture Notes in Computer Science, no. 2838. Springer Verlag, Belin, 2003, pp. 204-216.

[52] P. B.-S. Ralf Herbirch, Thore Graepel and K. Obermayer, "Learning preference relations for information retrieval," in Proceedings of the AAAI-98 Workshop on Learning for Text Categorization, 1998, pp. 83-86.

[53] J. Delgrande, H. Tompits, T. Schaub, and K. Wang, "A classification and survey of preference handling approaches in nonmonotonic reasoning," Computational Intelligence, vol. 20, no. 2, pp. 308334, April 2004.

[54] D. Herrmann, R. Wendolsky, and H. Federrath, "Website fingerprinting: attacking popular privacy enhancing technologies with the multinomial na\&\#239;ve-bayes classifier," in Proceedings of the 2009 ACM workshop on Cloud computing security, ser. CCSW '09. New York, NY, USA: ACM, 2009, pp. 31-42.

[55] S. Peddinti and N. Saxena, "On the privacy of web search based on query obfuscation: A case study of trackmenot," in Privacy Enhancing Technologies, ser. Lecture Notes in Computer Science, M. Atallah and N. Hopper, Eds. Springer Verlag Berlin, 2010, vol. 6205, pp. 19-37.

[56] E. Kushilevitz and R. Ostrovsky, "Replication is not needed: Single database, computationally-private information retrieval," in Proceedings of the 38th Annual Symposium on Foundations of Computer Science, FOCS '97, Miami Beach, Florida, USA, October 19-22, 1997. IEEE Computer Society, 1997, pp. 364-373.

[57] A. Papoulis, Probability, Random Variables, and Stochastic Processes, 3rd ed. McGraw-Hill Companies, 1991.

[58] E. T. Bell, "Exponential numbers," American Mathematical Monthly, vol. 41, pp. 411-419, 1934.

[59] N. G. de Bruijn, Asymptotic Methods in Analysis. Dover Publications, 1981.

[60] A. Nijenhuis and H. S. Wilf, Combinatorial Algorithms, 2nd ed., W. Rheinboldt, Ed. Academic Press Inc, 1978.

[61] L. Devroye, Non-Uniform Random Variate Generation. SpringerVerlag, New York, 1986.

[62] A. Shepitsen, J. Gemmell, B. Mobasher, and R. Burke, "Personalized recommendation in social tagging systems using hierarchical clustering," in RecSys '08: Proceedings of the 2008 ACM conference on Recommender systems. New York, NY, USA: ACM, 2008, pp. 259-266.

[63] R. Burke, "Knowledge-based recommender systems," in Encyclopedia of Library and Information Systems. 
[64] D. Kelly and J. Teevan, "Implicit feedback for inferring user preference: a bibliography," SIGIR Forum, vol. 37, no. 2, pp. 18-28, 2003.

[65] I. T. Jolliffe, Principal Component Analysis. Springer Verlag, 2002.

[66] F. Bacchus, A. J. Grove, J. Y. Halpern, and D. Koller, "From statistical knowledge bases to degrees of belief," The Computing Research Repository (CoRR), vol. cs.AI/0307056, 2003.

[67] C. Troncoso, B. Gierlichs, B. Preneel, and I. Verbauwhede, "Perfect matching disclosure attacks," in Privacy Enhancing Technologies, 8th International Symposium, PETS 2008, Leuven, Belgium, July 2325, 2008, Proceedings, ser. Lecture Notes in Computer Science, N. Borisov and I. Goldberg, Eds., vol. 5134. Springer Verlag, Berlin, 2008, pp. 2-23.

[68] R. M. Bell, Y. Koren, and C. Volinsky, "The BellKor solution to the Netflix prize," AT\&T Labs Research,, Technical Report, 2007.

[69] M. R. Jerrum, "The complexity of finding minimum-length generator sequences," Theoretical Computer Science, vol. 36, pp. 265-289, 1985.

[70] J. P. C. Vergara, "Sorting by bounded permutations," Ph.D. dissertation, Virginia Polytechnic Institute \& State University, 1998.

[71] T. Schiavinotto and T. Stützle, "A review of metrics on permutations for search landscape analysis," Computers and Operations Research, vol. 34, no. 10, pp. 3143-3153, October 2007.

\section{APPENDIX A \\ MATCHING IN PERMUTATIONS}

Lemma 3 (Uniformly random case) Consider a finite set $T$. If $p=\left(t_{1}, t_{2}, \ldots, t_{|T|}\right)$ is a permutation chosen uniformly at random from $M(T)$, then, for any given $\lambda \leq|T|$ and $T^{\prime} \subseteq$ $T$ such that $\left|T^{\prime}\right| \leq \lambda$, the probability that $\left\{t_{1}, t_{2}, \ldots, t_{\lambda}\right\} \supseteq$ $T^{\prime}$ is given by

$$
\Gamma_{\mathrm{u}}\left(T, \lambda, T^{\prime}\right)=\left(\begin{array}{c}
|T|-\left|T^{\prime}\right| \\
\lambda-\left|T^{\prime}\right|
\end{array}\right)\left(\begin{array}{c}
|T| \\
\lambda
\end{array}\right)^{-1} .
$$

Proof: Consider the task of selecting $p$ such that $\left\{t_{1}, t_{2}, \ldots, t_{\lambda}\right\} \supseteq T^{\prime}$. This task can be done by selecting $T^{\prime}$ as the first $\left|T^{\prime}\right|$ elements for $p$, then selecting the next $\lambda-\left|T^{\prime}\right|$ elements for $p$ from the remaining tags - there are $\left(\begin{array}{c}|T|-\left|T^{\prime}\right| \\ \lambda-\left|T^{\prime}\right|\end{array}\right)$ ways to do this — and, finally selecting the remaining elements in an arbitrary order. Since it is possible to permute the first $\lambda$ and the last $|T|-\lambda$ elements of $p$ without violating the requirement, there are $\left(\begin{array}{c}|T|-\left|T^{\prime}\right| \\ \lambda-\left|T^{\prime}\right|\end{array}\right) \lambda !\left(\lambda-\left|T^{\prime}\right|\right)$ ! permutations in $M(T)$ that satisfy the requirement. The result follows because each permutation has probability $(|T| !)^{-1}$ to be chosen as $p$.

Lemma 4 (Uniformly random case) Consider a finite set T. If $p=\left(t_{1}, t_{2}, \ldots, t_{|T|}\right)$ and $p^{\prime}=\left(t_{1}^{\prime}, t_{2}^{\prime}, \ldots t_{|T|}^{\prime}\right)$ are two permutations chosen independently and uniformly at random from $M(T)$, then, for any given $\lambda^{\prime}, \lambda$ such that $\lambda^{\prime} \leq \lambda \leq|T|$ and $T_{\Lambda} \subseteq T$, such that $\left|T_{\Lambda}\right|=\lambda^{\prime}$, the probabilities $\operatorname{Pr}\left(T\left(p, p^{\prime}, \lambda\right)=T_{\Lambda}\right)$ and $\operatorname{Pr}\left(\left|T\left(p, p^{\prime}, \lambda\right)\right|=\lambda^{\prime}\right)$ are given by

$$
\Phi_{\mathrm{u}}\left(|T|, \lambda, \lambda^{\prime}\right)=\left(\begin{array}{c}
|T|-\lambda \\
\lambda-\lambda^{\prime}
\end{array}\right)\left(\begin{array}{c}
|T|-\lambda^{\prime} \\
\lambda-\lambda^{\prime}
\end{array}\right)\left(\begin{array}{c}
|T| \\
\lambda
\end{array}\right)^{-2} \text { and }
$$

$$
\Phi_{\mathrm{u}}^{\prime}\left(|T|, \lambda, \lambda^{\prime}\right)=\left(\begin{array}{c}
\lambda \\
\lambda^{\prime}
\end{array}\right)\left(\begin{array}{c}
|T|-\lambda \\
\lambda-\lambda^{\prime}
\end{array}\right)\left(\begin{array}{c}
|T| \\
\lambda
\end{array}\right)^{-1} \text {, respectively. }
$$

Proof: We first show the correctness of Eq. (9). Suppose that $p$ is fixed and consider the task of selecting $p^{\prime}$ such that $T\left(p, p^{\prime}, \lambda\right)=T_{\Lambda}$. If $\left\{t_{1}, t_{2}, \ldots, t_{\lambda}\right\} \nsupseteq T_{\Lambda}$, then it is impossible to do this. Otherwise, the task can be done as follows. First select $T_{\Lambda}$ as the first $\lambda^{\prime}$ elements for $p^{\prime}$. Then select the next $\lambda-\lambda^{\prime}$ elements for $p^{\prime}$ from $\left\{t_{\lambda+1}, t_{\lambda+2}, \ldots, t_{|T|}\right\}$; there are $\left(\begin{array}{c}|T|-\lambda \\ \lambda-\lambda^{\prime}\end{array}\right)$ ways to do this. Finally, select the remaining tags for $p^{\prime}$ in an arbitrary order. Since it is possible to permute the first $\lambda$ and the last $|T|-\lambda$ elements of $p$ without violating the requirement, there are $\left(\begin{array}{c}|T|-\lambda \\ \lambda-\lambda^{\prime}\end{array}\right) \lambda !(|T|-\lambda)$ ! permutations in $M(T)$ that satisfy the requirement, and since each is chosen as $p$ with probability $(|T| !)^{-1}$, we have that $\operatorname{Pr}\left(\left(T\left(p, p^{\prime}, \lambda\right)=\right.\right.$ $\left.\left.T_{\Lambda}\right) \mid\left\{t_{1}, t_{2}, \ldots, t_{\lambda}\right\} \supseteq T_{\Lambda}\right)=\left(\begin{array}{c}|T|-\lambda \\ \lambda-\lambda^{\prime}\end{array}\right)\left(\begin{array}{c}|T| \\ \lambda^{\prime}\end{array}\right)^{-1}$. By Lemma 3, we have that $\operatorname{Pr}\left(\left\{t_{1}, t_{2}, \ldots, t_{\lambda}\right\} \supseteq T_{\Lambda}\right)=\left(\begin{array}{c}|T|-\left|T_{\Lambda}\right| \\ \lambda-\left|T_{\Lambda}\right|\end{array}\right)\left(\begin{array}{c}|T| \\ \lambda\end{array}\right)^{-1}$. Eq. (9) follows because $p$ and $p^{\prime}$ are chosen independently. Now we show the correctness of Eq. (10). Suppose that $p$ is fixed and consider the task of selecting $p^{\prime}$ such that $\left|T\left(p, p^{\prime}, \lambda\right)\right|=\lambda^{\prime}$ for a given $\lambda^{\prime} \leq \lambda$. This task can be performed by selecting the first $\lambda$ elements of $p^{\prime}$ by first choosing $\lambda^{\prime}$ elements from $\left\{t_{1}, t_{2}, \ldots, t_{\lambda}\right\}$ - there are $\left(\begin{array}{l}\lambda \\ \lambda^{\prime}\end{array}\right)$ ways to do this - and then selecting $\lambda-\lambda^{\prime}$ elements from $\left\{t_{\lambda+1}, t_{\lambda+2}, \ldots, t_{|T|}\right\}$ - there are $\left(\begin{array}{c}|T|-\lambda \\ \lambda-\lambda^{\prime}\end{array}\right)$ ways to do this. As a result, $\left|T\left(p, p^{\prime}, \lambda\right)\right|=\lambda^{\prime}$. The remaining $|T|-\lambda$ elements of $p^{\prime}$ are chosen in an arbitrary order. Since it is possible to permute the first $\lambda$ and the last $|T|-\lambda$ elements of $p^{\prime}$ without violating the requirement, there are $\left(\begin{array}{c}\lambda \\ \lambda^{\prime}\end{array}\right)\left(\begin{array}{c}|T|-\lambda \\ \lambda-\lambda^{\prime}\end{array}\right) \lambda !(|T|-\lambda)$ ! different permutations that satisfy the requirement. The result follows because every permutation has probability $(|T| !)^{-1}$ to be chosen as $p^{\prime}$.

Lemma 5 (Population case) Consider a finite set $T$, a population $P=\left\{p_{1}, p_{2}, \ldots, p_{|P|}\right\}$ of permutations of this set, and two permuations, denoted $p=\left(t_{1}, t_{2}, \ldots, t_{|T|}\right)$ and $p^{\prime}$, chosen from $P$ using simple random sampling with replacement. For any given $\lambda^{\prime}, \lambda$ such that $0 \leq \lambda^{\prime} \leq \lambda \leq|T|$, and $T^{\prime} \subseteq T$ such that $\left|T^{\prime}\right| \leq \lambda$, the probabilities $\operatorname{Pr}\left(\left\{t_{1}, t_{2}, \ldots, t_{\lambda}\right\} \supseteq T^{\prime}\right)$ and $\operatorname{Pr}\left(\left|T\left(p, p^{\prime}, \lambda\right)\right|=\lambda^{\prime}\right)$ are given by

$$
\Gamma_{\mathrm{p}}\left(P, \lambda, T^{\prime}\right)=\frac{\left|\left\{\left(t_{1}^{\prime}, \ldots, t_{|T|}^{\prime}\right) \in P:\left\{t_{1}^{\prime}, \ldots, t_{\lambda}^{\prime}\right\} \supseteq T^{\prime}\right\}\right|}{|P|}
$$

and

$$
\Phi_{\mathrm{p}}^{\prime}\left(P, \lambda, \lambda^{\prime}\right)=\frac{\left|\left\{\left(p, p^{\prime}\right) \in P^{2}:\left|T\left(p, p^{\prime}, \lambda\right)\right|=\lambda^{\prime}\right\}\right|}{|P|^{2}},
$$

respectively.

\section{APPENDIX B MATCHING IN RANDOM PARTITIONS}

Lemma 6 The probability that a $k$-tuple of elements of a finite set of size $n$ belongs to the same equivalence class of a uniformly at random chosen partition (equivalence relation) of that set, is given by $\Pi(n, k)=B_{n-k+1} / B_{n}$, where $B_{n}$ denotes nth Bell number [58].

Proof: Let $S$ denote the set and let $K \subseteq S$ denote the $k$-tuple. Since the set $S^{\prime}=(S \backslash K) \cup\{K\}$ that is 
obtained by merging the $k$-tuple into a single element has cardinality $\left|S^{\prime}\right|=n-k+1$, the number of its set partitions is $B_{n-k+1}$, and the result follows.

\section{APPEndix C PROOFS}

\section{C.1 Proof of Lemma 1}

Let $p=\left(t_{1}, t_{2}, \ldots, t_{|T|}\right)$ denote the 'origin' preference of both $p_{i}^{\prime}$ and $p_{i^{\prime}}^{\prime}$. Suppose that $p$ is fixed and consider the task of selecting the preference $p_{i}^{\prime}=\left(t_{1}^{\prime}, t_{2}^{\prime}, \ldots, t_{|T|}^{\prime}\right)$ in a manner compatible with $\sigma_{\omega^{(\kappa, \lambda)}}(p)$ and such that $\left\{t_{1}^{\prime}, t_{2}^{\prime}, \ldots, t_{\kappa}^{\prime}\right\} \supseteq T^{\prime}$ for a given $T^{\prime} \subseteq T$ with $\left|T^{\prime}\right| \leq \kappa$. If $\left\{t_{1}, t_{2}, \ldots, t_{\lambda}\right\} \nsupseteq T^{\prime}$, then it is impossible to do this. Otherwise, it can be done as follows. First select $T^{\prime}$ as the first $\left|T^{\prime}\right|$ elements for $p_{i}^{\prime}$. Then select $\kappa-\left|T^{\prime}\right|$ elements from the first $\lambda$ elements of $p$ for $p_{i}^{\prime}$, such that none of them is identical to any element in $T^{\prime}$; there exist exactly $\left(\begin{array}{c}\lambda-\left|T^{\prime}\right| \\ \kappa-\left|T^{\prime}\right|\end{array}\right)$ ways to do this. Finally, select the remaining elements for $p_{i}^{\prime}$ in an arbitrary order. The number of $\sigma_{\omega(\kappa, \lambda)}$ 's outputs that satisfy the requirement is therefore $\left(\begin{array}{c}\lambda-\left|T^{\prime}\right| \\ \kappa-\mid T^{\prime}\end{array}\right) \kappa !(|T|-\kappa) !$, and since each output has probability $\frac{(\lambda-\kappa) !}{\lambda !(|T|-\kappa) !}$ (see Eq. (1)), we have that

$$
\operatorname{Pr}\left(\left\{t_{1}^{\prime}, \ldots, t_{\kappa}^{\prime}\right\} \supseteq T^{\prime} \mid\left\{t_{1}, \ldots, t_{\lambda}\right\} \supseteq T^{\prime}\right)=\left(\begin{array}{l}
\lambda-\left|T^{\prime}\right| \\
\kappa-\left|T^{\prime}\right|
\end{array}\right)\left(\begin{array}{l}
\lambda \\
\kappa
\end{array}\right)^{-1} .
$$

Now suppose that $\left\{t_{1}^{\prime}, t_{2}^{\prime}, \ldots, t_{\kappa}^{\prime}\right\} \supseteq T^{\prime}$, and consider selecting the preference $p_{i^{\prime}}^{\prime}=\left(t_{1}^{\prime \prime}, t_{2}^{\prime \prime}, \ldots, t_{|T|}^{\prime \prime}\right)$ in a manner compatible with $\sigma_{\omega^{(\kappa, \lambda)}}(p)$ and such that $T\left(p_{i}^{\prime}, p_{i^{\prime}}^{\prime}, \kappa\right)=$ $T^{\prime}$. This task can be done as follows. First select $T^{\prime}$ as the first $\left|T^{\prime}\right|$ elements for $p_{i^{\prime}}^{\prime}$. Then select $\kappa-\left|T^{\prime}\right|$ elements from the first $\lambda$ elements of $p$ for $p_{i^{\prime}}^{\prime}$, such that none of them is identical to any element in the first $\kappa$ elements of $p_{i}^{\prime}$; there exist exactly $\left(\begin{array}{c}\lambda-\kappa \\ \kappa-\mid T^{\prime}\end{array}\right)$ different ways to do this. Finally, select the remaining elements for $p_{i^{\prime}}^{\prime}$ in an arbitrary order. The number of $\sigma_{\omega^{(\kappa, \lambda)}}$ 's outputs that satisfy the requirement is therefore $\left(\begin{array}{c}\lambda-\kappa \\ \kappa-\left|T^{\prime}\right|\end{array}\right) \kappa !(|T|-\kappa) !$ and since these outputs have probability $\frac{(\lambda-\kappa) !}{\lambda !(|T|-\kappa) !}$ (see Eq. (1)), we have that

$$
\operatorname{Pr}\left(T\left(p_{i}^{\prime}, p_{i^{\prime}}^{\prime}, \kappa\right)=T^{\prime} \mid\left\{t_{1}^{\prime}, \ldots, t_{|\kappa|}^{\prime}\right\} \supseteq T^{\prime}\right)=\left(\begin{array}{c}
\lambda-\kappa \\
\kappa-\left|T^{\prime}\right|
\end{array}\right)\left(\begin{array}{c}
\lambda \\
\kappa
\end{array}\right)^{-1} .
$$

Since $p$ is selected uniformly at random from $M(T)$, by Lemma 3, we have that

$$
\operatorname{Pr}\left(\left\{t_{1}, t_{2}, \ldots, t_{\lambda}\right\} \supseteq T^{\prime}\right)=\left(\begin{array}{c}
|T|-\left|T^{\prime}\right| \\
\lambda-\left|T^{\prime}\right|
\end{array}\right)\left(\begin{array}{c}
|T| \\
\lambda
\end{array}\right)^{-1} .
$$

Eq. (3) now follows from Eq. (13) (14), and (15). In order to arrive at Eq. (4), on the other hand, observe that, given the specification of $\sigma_{\omega^{(\kappa, \lambda)}}$ (see Eq. (1)) and the assumption that $i \not i^{\prime}$, which implies that $p_{o(i)}$ and $p_{o\left(i^{\prime}\right)}^{\prime}$ are selected independently and uniformly at random from $M(T)$, both $p_{i}^{\prime}$ and $p_{i^{\prime}}^{\prime}$ may take the value of any preference in $M(T)$ with equal probability. Hence, Lemma 4 and, in particular, Eq. (9) applies. Eq. (4) follows.

\section{C.2 Proof of Lemma 2}

Proof: Assume that $i \sim i^{\prime}$ and let $p=\left(t_{1}, t_{2}, \ldots, t_{|T|}\right)$ denote the 'origin' preference of both $p_{i}^{\prime}$ and $p_{i^{\prime}}^{\prime}$, and let $p_{i}^{\prime}=\left(t_{1}^{\prime}, t_{2}^{\prime}, \ldots, t_{|T|}^{\prime}\right)$. Eq. (5) follows from Eq. (13) and (14) and the fact that $\operatorname{Pr}\left(\left\{t_{1}, t_{2}, \ldots, t_{\lambda}\right\} \supseteq T^{\prime}\right)=$ $\Gamma_{\mathrm{p}}\left(P, \lambda, T^{\prime}\right)$, where $\Gamma_{\mathrm{p}}(\cdot, \cdot, \cdot)$ is given by Eq. (11). Now assume that $i \nsim i^{\prime}$, let $p_{i}^{\prime}=\left(t_{1}^{\prime}, t_{2}^{\prime}, \ldots, t_{|T|}^{\prime}\right)$, and let $A_{T_{c}}$ denote the event that $\left\{t_{1}^{\prime}, t_{2}^{\prime}, \ldots, t_{\kappa}^{\prime}\right\}-T_{c}=T^{\prime}$ for some $T_{c} \subseteq T-T^{\prime}$. Suppose that $A_{T_{c}}$ takes place and consider the task of selecting $p_{i^{\prime}}^{\prime}$ in a manner compatible with $\sigma_{\omega(\kappa, \lambda)}\left(p_{o\left(i^{\prime}\right)}\right)$ and such that $T\left(p_{i}^{\prime}, p_{i^{\prime}}^{\prime}, \kappa\right) \supseteq T^{\prime}$. If $\left\{\tilde{t}_{1}, \tilde{t}_{2}, \ldots, \tilde{t}_{\lambda}\right\} \nsupseteq T^{\prime}$, then it is impossible to do this task. Otherwise it can be done as follows. First select $T^{\prime}$ as the first $\left|T^{\prime}\right|$ tags for $p_{i^{\prime}}^{\prime}$. Then select the next $\kappa-\left|T^{\prime}\right|$ tags from the first $\lambda$ tags of $p_{o\left(i^{\prime}\right)}$ such that none of them is identical to any tag in $T^{\prime}$; there exist exactly $\left(\begin{array}{l}\lambda-\left|T^{\prime}\right| \\ \kappa-\mid T^{\prime}\end{array}\right)$ ways to do this. Finally, select the remaining tags for $p_{i^{\prime}}^{\prime}$ in an arbitrary order. The number of $\sigma_{\omega^{(\kappa, \lambda)}}$ 's outputs that satisfy the requirement is therefore $\left(\begin{array}{l}\lambda-\left|T^{\prime}\right| \\ \kappa-\mid T^{\prime}\end{array}\right) \kappa !(|T|-\kappa) !$ and since each output has probability $\frac{(\lambda-\kappa) !}{\lambda !(|T|-\kappa) !}$ (see Eq. (1)), we have that $\operatorname{Pr}\left(T\left(p_{i}^{\prime}, p_{i^{\prime}}^{\prime}, \kappa\right) \supseteq T^{\prime} \mid A_{T_{c}}\right)=$ $\Gamma_{\mathrm{p}}\left(P, \lambda, T^{\prime}\right)\left(\begin{array}{l}\lambda-\left|T^{\prime}\right| \\ \kappa-\mid T^{\prime}\end{array}\right)\left(\begin{array}{l}\lambda \\ \kappa\end{array}\right)^{-1}$. From this follows that

$$
\begin{aligned}
& \operatorname{Pr}\left(T\left(p_{i}^{\prime}, p_{i^{\prime}}^{\prime}, \kappa\right)=T^{\prime} \mid A_{T_{c}}\right) \\
& \quad=\left(\begin{array}{l}
\lambda \\
\kappa
\end{array}\right)-1 \sum_{T_{x} \subseteq T_{c}}(-1)^{\left|T_{x}\right|} \Gamma_{\mathrm{p}}\left(P, \lambda, T_{x} \cup T^{\prime}\right)\left(\begin{array}{c}
\lambda-\left|T^{\prime}\right|-\left|T_{x}\right| \\
\kappa-\left|T^{\prime}\right|
\end{array}\right),
\end{aligned}
$$

$$
\begin{aligned}
& \text { and Eq. (6) follows because } \operatorname{Pr}\left(A_{T_{c}}\right)= \\
& \left(\begin{array}{l}
\lambda \\
\kappa
\end{array}\right)^{-1} \sum_{T_{c} \subseteq T-T^{\prime}} \Gamma_{\mathrm{p}}\left(P, \lambda, T_{c} \cup T^{\prime}\right) \\
& \left|T_{c}\right|=\kappa-\left|T^{\prime}\right|
\end{aligned}
$$

Dr. Andreas Pashalidis is a researcher at COSIC, Katholieke Universiteit Leuven in Belgium, with a focus on both theoretical and practical aspects of privacy and information security. Prior to joining COSIC, he worked for Siemens and NEC where he was active in standardization and contributed to numerous consulting and research projects.

Prof. Bart Preneel is a member of the COSIC research group at the Katholieke Universiteit Leuven in Belgium. He has authored more than 400 scientific publications and is inventor of 3 patents. His main research interests are cryptography and information security (e.g., privacy and evoting) and he frequently consults on these topics. He is president of the IACR (International Association for Cryptologic Research). He has served as program chair of 14 conferences and he has been invited speaker at more than 70 conferences in 30 countries. 